\title{
INFLUÊNCIA DA CLASSE DIAMÉTRICA NAS PROPRIEDADES FÍSICAS DA MADEIRA DE PARICÁ (Schizolobium parahyba var. amazonicum Huber ex Ducke)
}

Influence of the diametric class on the physical wood properties of paricá (Schizolobium parahyba var. amazonicum Huber ex Ducke)

Influencia de la clase diamétrica sobre las propiedades físicas de la madera de paricá (Schizolobium parahyba var. amazonicum Huber ex Ducke)

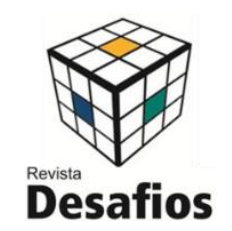

\section{Amália Carolina de Morais Gonçalves ${ }^{1}$, Adriano Reis Prazeres Mascarenhas*1, Rafael Rodolfo de Melo $^{2}$ \\ ${ }^{1}$ Universidade Federal de Rondônia - UNIR, Departamento Acadêmico de Engenharia Florestal, Rolim de Moura, RO, Brasil.}

${ }^{2}$ Universidade Federal Rural do Semi-Árido - UFERSA, Departamento de Ciências Agronômicas e Florestais, Mossoró, RN, Brasil.

*Correspondência: Departamento Acadêmico de Engenharia Florestal, Av. Norte Sul, n. 7300, Bairro Nova Morada, Rolim de Moura, RO, Brasil.CEP: 76.940-000,e-mail adriano.mascarenhas@unir.br.

Artigo recebido em 25/07/2019 aprovado em 13/07/2020 publicado em 31/10/2020.

\section{RESUMO}

O cultivo de espécies florestais nativas incrementa o estoque de madeira no mercado e pode reduzir a pressão sobre florestas naturais. O paricá (Schizolobium parahyba var. amazonicum Huber ex Ducke) possui características compatíveis ao monocultivo e sua madeira desperta interesse comercial, mas as propriedades da madeira de paricá em plantios é pouco estudada. Objetivou-se estudar a influência das classes diamétricas na variabilidade das propriedades físicas da madeira jovem de paricá em plantio comercial. Abateram-se 20 árvores distribuídas em cinco classes diamétricas para determinação da densidade básica $(\mathrm{db})$; porosidade $(\Phi)$; contrações: longitudinal $(\varepsilon l)$, radial ( $\varepsilon r)$, tangencial ( $\varepsilon t)$ e volumétrica $(\Delta \mathrm{v})$; e coeficiente de anisotropia (T/R). A densidade da madeira de paricá variou de 0,22 a $0,37 \mathrm{~g} / \mathrm{cm}^{3}$ e mostra-se crescente à medida que o diâmetro aumenta. A madeira foi classificada como leve e de alta porosidade (77 a 85\%). Os valores de $\varepsilon \mathrm{l}(0,22$ a $0,39 \%)$, $\varepsilon \mathrm{r}(1,74$ a $2,72 \%)$, $\varepsilon \mathrm{t}(3,14$ a $4,14 \%)$ e $\Delta \mathrm{v}(4,98$ a $6,64 \%)$ variaram entre as classes diamétricas e indicaram tendência de redução da estabilidade dimensional em árvores com maior diâmetro. Apesar disso, a madeira estudada é pouco retrátil e os valores de T/R $(1,59$ a 2,61\%) foram menores nas árvores de maior diâmetro.

Palavras-chave: Densidade da madeira; Física da madeira; Madeira Tropical.

\section{ABSTRACT}

Cultivation of native forest species increases the stock of wood on the market and reduce pressure on natural forests. Paricá (Schizolobium parahyba var. amazonicum Huber ex Ducke) has compatibilty for monoculture crop and its wood arouses commercial interest, but as a characteristic of wood from forest planting and little studied. This work aimed study influence of diametric classes on variability of properties of young paricá wood in commercial planting. 20 trees were harvested, distributed in five diametric classes to determine the basic density (db); porosity $(\Phi)$;

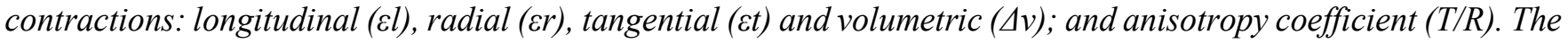
density of the wood in particular ranges from 0.22 to $0.37 \mathrm{~g} / \mathrm{cm}^{3}$ and shows an increasing increase as the diameter increases. Wood was classified as light and highly porosity (77 to 85\%). The values of $\varepsilon l(0.22$ to $0.39 \%)$, $8 \mathrm{r}(1.74$ to $2.72 \%)$, et (3.14 to $4.14 \%)$ and $\Delta v(4.98$ to $6.64 \%)$ varies between diametric classes and indicates a tendency to reduce dimensional stability in trees with larger diameter. Despite this, a studied wood is not very retractable and the $T / R$ values (1.59 to $2.61 \%$ ) were lower in trees with a larger diameter. 
Keywords: Wood density; Wood physics; Tropical Wood.

\section{RESUMEN}

El cultivo de espécies florestais nativas aumenta o estoque de madeira no mercado e pode reduzir la presion sobre florestas naturais. Paricá (Schizolobium parahyba var. Amazonicum Huber ex Ducke) tiene características compatibles con el monocultivo y su madera despierta interés comercial, pero las propiedades de la madera de paricá en las plantaciones están poco estudiadas. El objetivo fue estudiar la influencia de las clases diametrales en la variabilidad de las propiedades físicas de la madera joven de paricá en la plantación comercial. Se talaron 20 árboles, distribuidos en cinco clases diametrales para la determinación de la densidad básica (db); porosidade

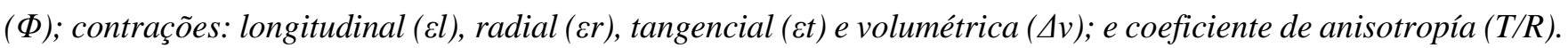
La densidad de la madera de paricá varió de 0,22 a $0,37 \mathrm{~g} / \mathrm{cm}^{3}$ y aumenta a medida que aumenta el diámetro. La madera se clasificó como ligera y de alta porosidad (77 a 85\%). Los valores de $\varepsilon l(0,22$ a 0,39\%), er (1,74 a 2,72\%), Et $(3,14$ a 4,14\%) y $\Delta v(4,98$ a 6,64\%) variaron entre las clases diametrales e indicaron una tendencia a reducir la estabilidad dimensional en árboles con un diámetro mayor. A pesar de esto, la madera estudiada no es muy retráctil y los valores de T/R (1,59 a 2,61\%) fueron más bajos en árboles con un diámetro mayor.

Descriptores: Densidad de la madera; Física de la madera; Madera Tropical.

\section{INTRODUÇÃO}

A região Amazônica destaca-se na produção de madeiras tropicais no Brasil. No entanto, devido a exploração ilegal de florestas nativas houve redução na disponibilidade de matéria-prima no mercado (REIS et al., 2019).

Com isso, houve intensificação da fiscalização ambiental que desestimulou atividades ilícitas de exploração florestal. Isso contribuiu para o setor público estabelecer parâmetros de boas práticas para obtenção de madeira por meio de metodologias de exploração com impacto reduzido (SALES et al., 2019).

Entretanto, apenas ações dessa natureza não têm sido suficientes para aumentar a disponibilidade de madeira nativa. Uma alternativa para atender essa demanda é o incentivo aos plantios florestais. (VERÍSSIMO e PEREIRA, 2014).

As florestas plantadas correspondem a mais de $90 \%$ de toda a madeira produzida para fins industriais e são responsáveis por $6,9 \%$ do PIB industrial no Brasil (IBÁ, 2019).

A maioria desses plantios são constituídos por espécies exóticas, geralmente, dos gêneros Eucalyptus e Pinus, devido ao rápido crescimento e adaptabilidade em povoamentos homogêneos (STEFANO et al.,
2019). Apesar disso, os povoamentos comerciais no Brasil podem ser incrementados em produção, estoque de matéria prima e diversificação com o cultivo de florestas utilizando-se espécies nativas.

O paricá (Schizolobium parahyba var. amazonicum Huber ex Ducke) insere-se nesse contexto, pois sua madeira é utilizada na produção de laminas e painéis engenheirados (MELO et al., 2020). A área plantada de paricá no Brasil apresenta pequeno crescimento, no entanto, é constante principalmente na região Amazônica (IBÁ, 2019). Sua adaptação e o rápido crescimento possibilitam utilizar sua madeira ainda jovem, já que a realização de desbastes em ciclos de curta rotação pode apresentar viabilidade (VIDAURRE et al., 2018).

Essas características aumentam o potencial dessa espécie para atendimento da demanda por matéria-prima a partir de plantios comerciais (MIRANDA et al., 2016; SILVA et al., 2020). Mesmo assim, informações a respeito das propriedades tecnológicas da madeira de paricá em povoamentos comerciais são escassas. Então, para subsidiar a indústria e silvicultores com informações a respeito do cultivo desta espécie, estudos nesse sentido são fundamentais. 
Isso porque as características da madeira variam entre espécies, entre árvores de mesma espécie, em função da qualidade de sítio, tratamentos silviculturais e do material genético (EUFRADEJUNIOR et al., 2017; MOORE et al., 2017). Com os parâmetros tecnológicos da madeira é possível dimensionar o material de acordo com as aplicações finais, visando a maior durabilidade, estabilidade e resistência (TRUGILHO et al., 2014; RAMAGE et al., 2017).

Com base nesse cenário, objetivou-se estudar a influência das classes diamétricas na variabilidade das propriedades físicas da madeira jovem de paricá em plantio comercial na Amazônia.

\section{MATERIAIS E MÉTODOS}

\section{Caracterização da área de estudo}

A madeira utilizada nesse trabalho foi obtida de um plantio comercial de paricá (Schizolobium parahyba var. amazonicum Huber ex Ducke). A área localiza-se às margens da rodovia estadual RO-010 no município de Rolim de Moura, Rondônia, Brasil (Figura 1).

Figura 1. Mapa de localização do plantio de paricá (Schizolobium parahyba var. amazonicum Huber ex Ducke) em Rolim de Moura, Rondônia, Brasil.
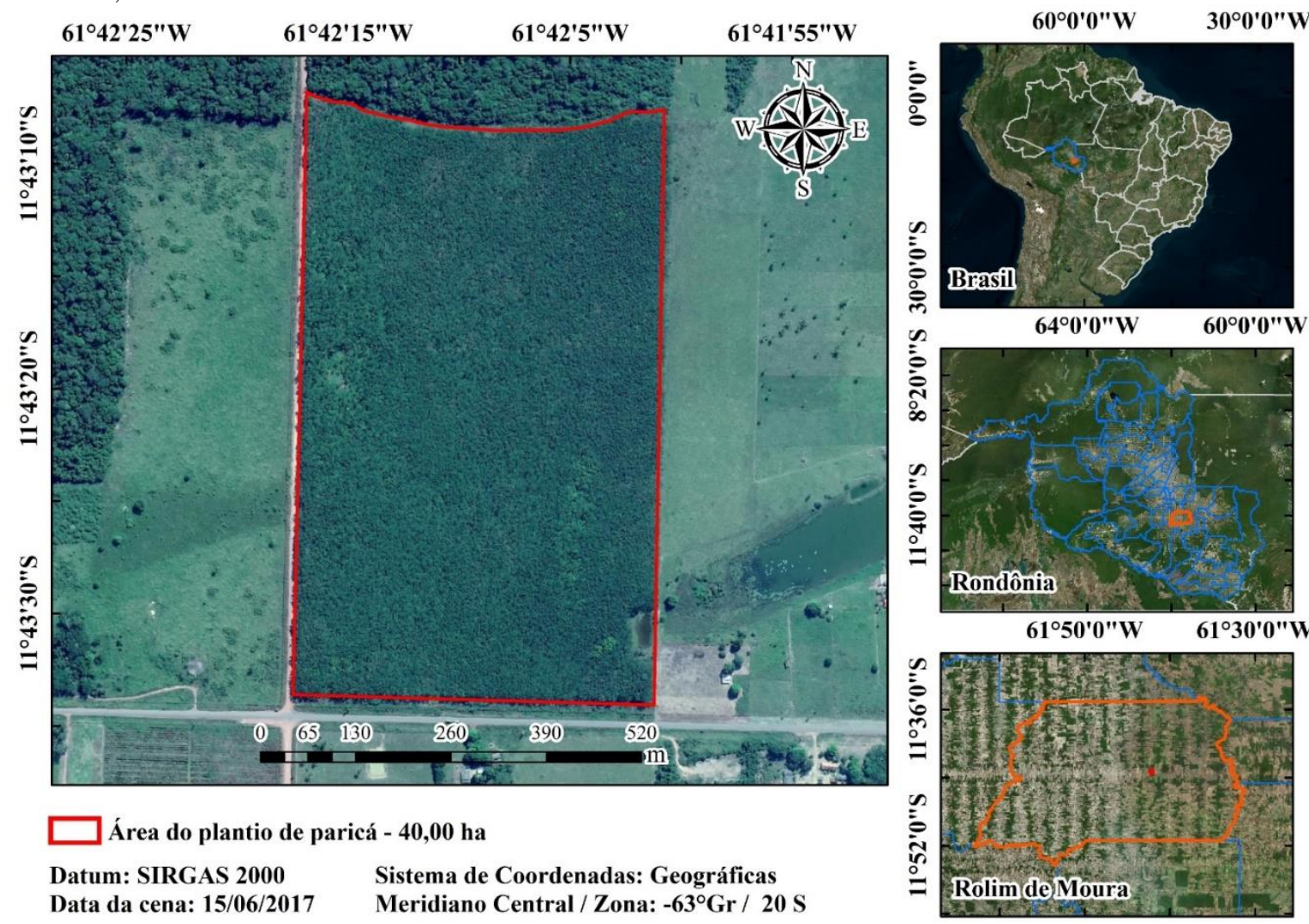

O clima da região é do tipo Am (tropical de monções), a temperatura média varia entre $24{ }^{\circ} \mathrm{C}$ a $26^{\circ} \mathrm{C}$ e a precipitação anual é de $2.200 \mathrm{~mm}$ a $2.500 \mathrm{~mm}$ (ALVARES et al., 2014). A altitude da região está entre $200 \mathrm{~m}$ a $300 \mathrm{~m}$ e o solo é do tipo Latossolo vermelho-escuro eutrófico com ocorrência de
Latossolo vermelho-amarelo distrófico (SIPAM, 2006).

O plantio foi implantado no ano de 2012, abrangendo uma área de, aproximadamente, 40 ha, no espaçamento $3 \times 3 \mathrm{~m}$. Nos três primeiros anos de cultivo, foram realizados tratos silviculturais (desrama 
e roçadas) e no quarto ano realizou-se desbaste seletivo na área leste do plantio com a remoção das árvores suprimidas.

\section{Seleção de árvores e amostragem da madeira}

Conduziu-se um inventário no povoamento delimitando-se 8 unidades amostrais de 20 x $100 \mathrm{~m}$, respeitando uma distância da borda de 50 m, tomandose os valores de diâmetro a altura do peito (DAP).

A partir desta coleta as árvores levantadas foram agrupadas em cinco classes diamétricas (Classe I - 6,2 a 10,6 cm, com 141 árvores; Classe II - 10,6 a 15,0 cm, com 493 árvores; Classe III - 15,0 a 19,4 cm, com 251 árvores; Classe IV - 19,4 a 23,8 cm, com 29 árvores; Classe V - 23,8 a 28,2 cm, com 2 árvores).

Em seguida, para cada classe de DAP, foram abatidas quatro árvores, conforme ASTM D5536 (ASTM, 2017), utilizando-se como critério de seleção o centro de classe $\pm 1 \mathrm{~cm}$. Para cada árvore abatida foram retirados toretes com aproximadamente $100 \mathrm{~cm}$ de comprimento a partir da base, utilizando-se serra motorizada.

\section{Caracterização física da madeira de paricá}

Realizou-se o desdobro primário dos toretes em serra de fita vertical, obtendo-se pranchões centrais variando de $5 \mathrm{~cm}$ a $25 \mathrm{~cm}$ de largura, $4 \mathrm{~cm}$ de espessura e $100 \mathrm{~cm}$ de comprimento. Em seguida os pranchões foram submetidos ao desdobro secundário utilizandose serras circulares para a confecção de corpos de prova com dimensões de 2,5 x 2,5 x 10,0 cm (largura $\mathrm{x}$ altura $\mathrm{x}$ comprimento).

Foram obtidos 10 corpos de prova por árvore, livres de defeitos (empenamento, rachaduras, perfurações e nós) e resquícios de medula ou casca.

As amostras foram submetidas à saturação em água até que atingissem massas constantes. Nesse momento mensurou-se as dimensões nos sentidos longitudinal, radial e tangencial com paquímetro digital $(0,01 \mathrm{~mm})$. Em seguida, as amostras foram submetidas a secagem em estufa com circulação e renovação de ar em temperatura de $105^{\circ} \mathrm{C} \pm 5^{\circ} \mathrm{C}$ e, após atingirem a condição anidra, tiveram suas massas e dimensões novamente medidas. Ao final, a madeira foi acondicionada em câmara climatizada em temperatura em $25^{\circ} \mathrm{C}$ e umidade de $65 \%$, na qual permaneceram até atingir umidade de equilíbrio em torno de $12 \%$.

Com os dados obtidos nas diferentes condições de umidade, foram determinadas as propriedades físicas: densidade básica $(\mathrm{db})$, contração longitudinal ( $\varepsilon 1)$, contração radial ( $\varepsilon r)$, contração tangencial (ct), contração volumétrica $(\Delta \mathrm{v})$, coeficiente de anisotropia (T/R) e porosidade $(\Phi)$, conforme os parâmetros descritos na norma ASTM D143 (ASTM, 2014).

\section{Análise dos resultados}

Os dados obtidos foram analisados por meio do teste de Fisher $(\mathrm{p}<0,01)$. Em seguida, ao se observar diferenças entre os pares de média, aplicou-se a análise de regressão, de modo que fosse diagnosticada a influência das classes diamétricas nas propriedades físicas da madeira.

\section{RESULTADOS E DISCUSSÃO}

De modo geral, relação entre a densidade foi e os diâmetros foi diretamente proporcional, com uma diferença de 44,83\% entre a Classe I e Classe V. Já a diferença de valores entre as classes diamétricas I e V foi de $32 \%$ (Figura 2).

Figura 2. Densidade básica $(\mathrm{db})$ da madeira de paricá (Schizolobium parahyba var. amazonicum Huber ex Ducke) em função da classe diamétrica. 


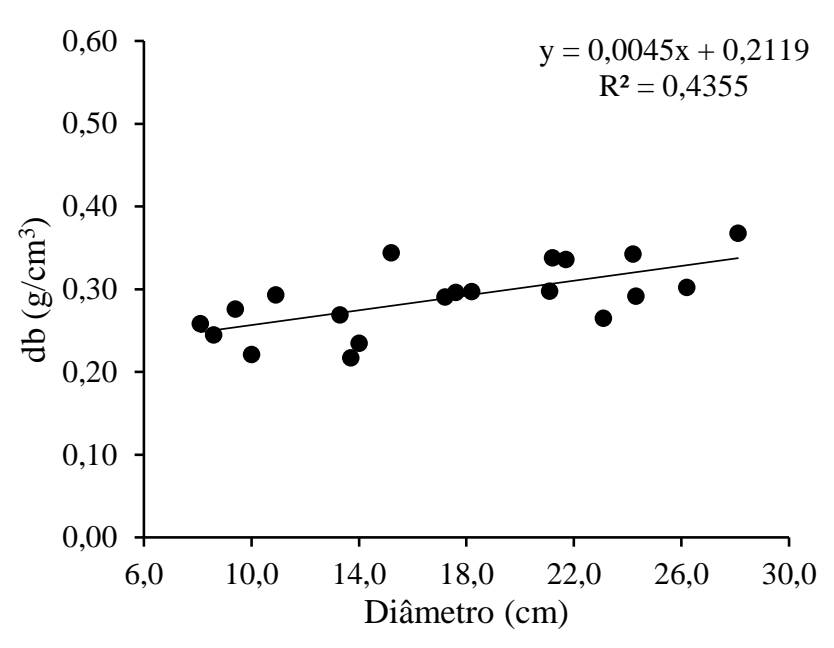

A explicação para estes resultados está positiva com o diâmetro, ou seja tendem a apresentar valores crescentes da medula em direção ao câmbio. De forma geral, os valores de densidade básica foram semelhantes aos obtidos em plantios de paricá estudados por Terezo et al., (2015) $\left(0,33 \mathrm{~g} / \mathrm{cm}^{3}\right)$ e Silva et al. (2020) $\left(0,25 \mathrm{~g} / \mathrm{cm}^{3}\right)$.

Em relação aos valores de $\Phi$ (Figura 3), os valores apresentaram-se dentro do esperado para a espécie em todas as classes diamétricas, classificandose como altamente porosa (MODES et al., 2018; LPF, 2018; MELO et al., 2020).

relacionada ao aumento da quantidade de lenho tardio em relação ao lenho juvenil no crescimento em diâmetro. Contribuindo para o entendimento desse raciocínio. Melo et al. (2014) e Modes (2016), ao estudarem a madeira de paricá, mencionaram que a densidade básica e aparente apresentam alta correlação

Figura 3. Porosidade $(\Phi)$ da madeira jovem de paricá (Schizolobium parahyba var. amazonicum Huber ex Ducke) em função da classe diamétrica.

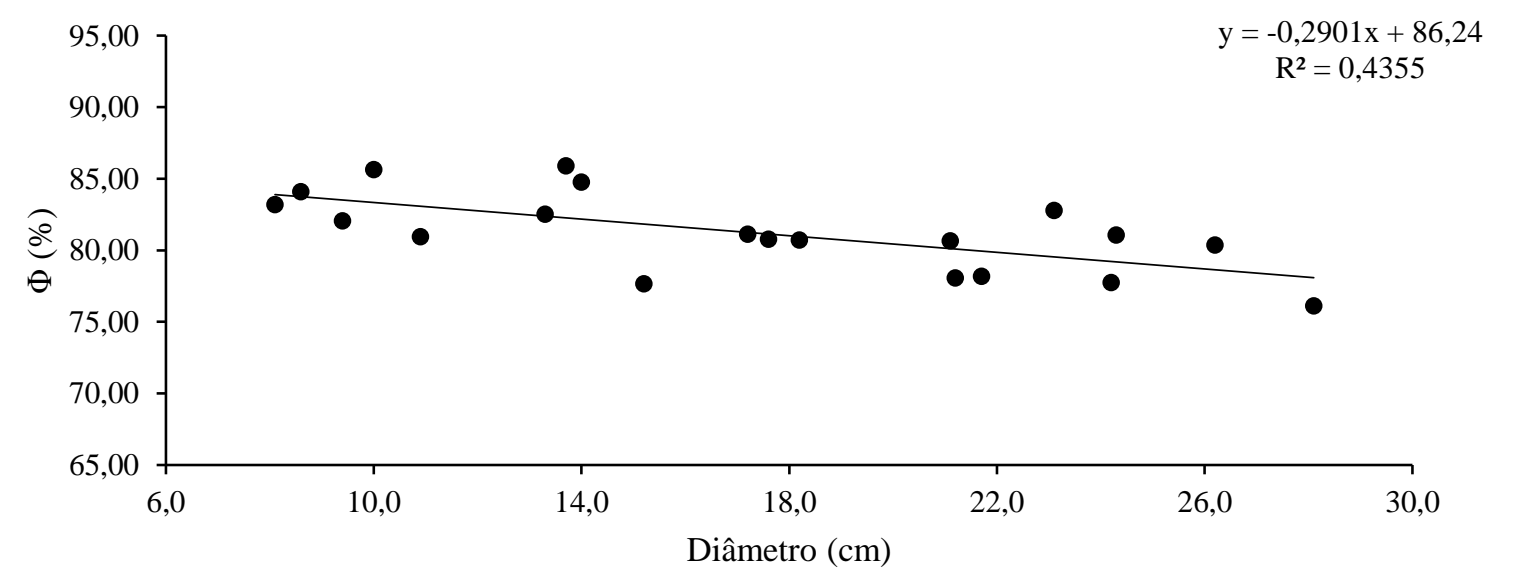

A porosidade é inversamente proporcional à densidade, devido a isso o material estudado foi classificado como de baixa densidade, pois todas as médias resultaram em valores menores que $0,50 \mathrm{~g} \mathrm{~cm}^{-}$ 3 (CARVALHO, 1996). Essa característica é muito importante na confecção de painéis laminados, porque madeiras com alta porosidade facilitam o processo de adesão entre lâminas devido à maior eficiência na umectação e penetração do adesivo (IWAKIRI et al, 2018).
Em relação as contrações lineares (longitudinal, radial e tangencial) e volumétrica (Figura 4), verificou-se que a tendência apresentada para o comportamento foi variável, dependendo do eixo avaliado. Para contração $\varepsilon l$, houve decréscimo nas classes intermediárias, sendo que os maiores valores foram observados para as árvores com menores e maiores diâmetros. Já para cr, verificou-se aumento progressivo com o incremento do diâmetro. 
Figura 4. Contrações longitudinal $(\varepsilon l)$, radial $(\varepsilon r)$, tangencial $(\varepsilon t)$, volumétrica $(\Delta \mathrm{v})$ da madeira de paricá (Schizolobium parahyba var. amazonicum Huber ex Ducke) em função da classe diamétrica.
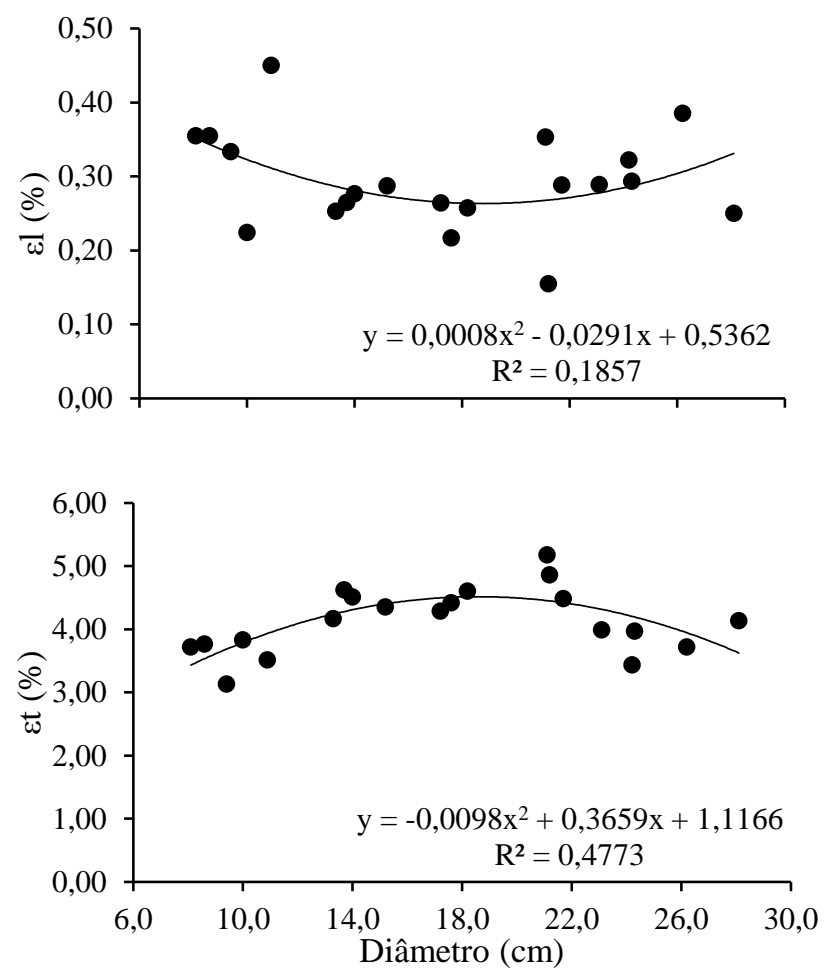

O motivo das maiores contrações radias terem sido visualizadas nas maiores classes diamétricas está ligado ao fato de que quanto maior o diâmetro maior a heterogeneidade da madeira em relação a proporção entre lenho tardio e lenho juvenil, características que, reconhecidamente, influenciam a estabilidade dimensional do material (MELO et al., 2013).

Para a contração no eixo tangencial ( $\varepsilon t$ ), foram observados maiores valores para as classes intermediárias, havendo um decréscimo do parâmetro para as árvores de maior e menor diâmetro, comportamento distinto do observado para os demais eixos.

Outro ponto a se destacar é que as contrações lineares evidenciam a natureza anisotrópica da madeira, conforme a descrição apresentada por Melo et al. (2010). Os autores explicaram que a contração longitudinal é mínima e a radial é menor que a obtida no sentido tangencial, sendo esta a mais importante
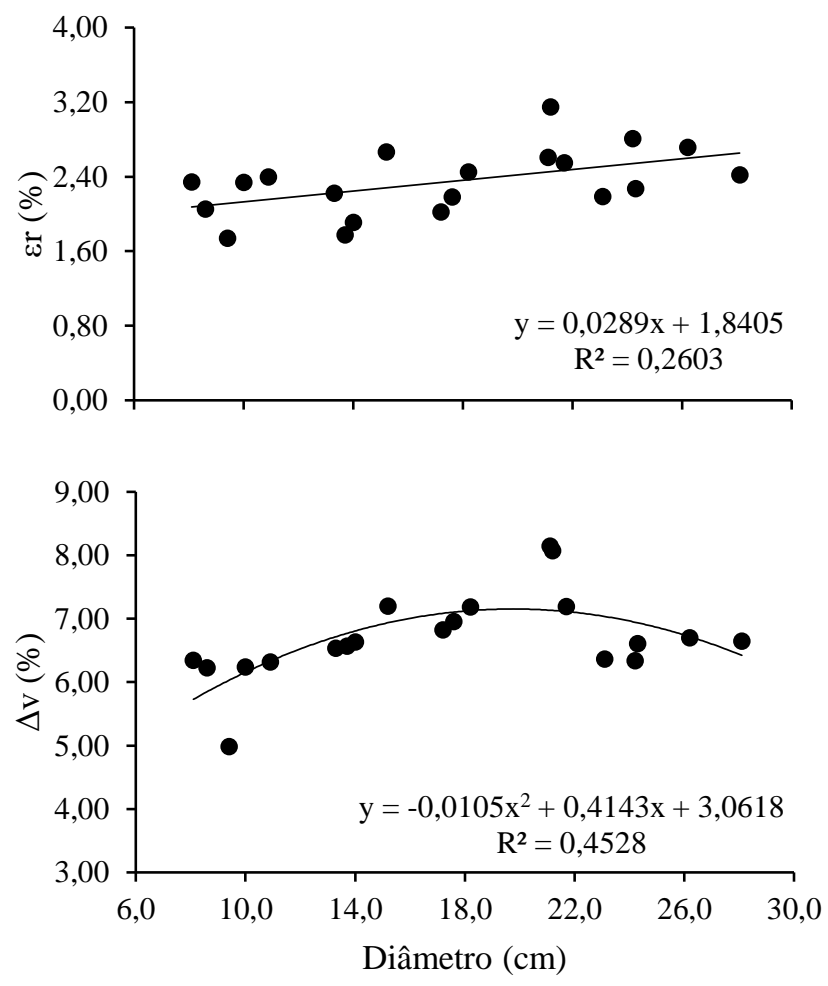

para fins práticos de utilização da madeira maciça. Quando as contrações tangenciais são muito intensas, existe possibilidade de ocorrência de rachaduras em lâminas e encanoamento de tábuas.

Quanto aos resultados de $\Delta \mathrm{v}$, verificou-se a mesma tendência observada para contração linear no eixo tangencial. Os valores observados para este parâmetro variaram entre $4,98 \%$ e $8,15 \%$. Carvalho (1996) estabeleceu uma classificação da madeira em relação a estabilidade dimensional, na qual considerase pouco retrátil quando a contração volumétrica é entre $5 \%$ e $10 \%$, ou ainda, quando as contrações tangencial e radial são menores que $7 \%$ e $4 \%$, nessa ordem, características similares as observadas no presente estudo.

Em linhas gerais, os valores para as contrações estão dentro do esperado para a espécie. Mascarenhas (2015) estudando a madeira de paricá com 19 anos de idade produzida em sistema agroflorestal na 
Amazônia, obteve valores de $\varepsilon l$, $\varepsilon$ r e $\varepsilon t$ na ordem de $0,38 \%, 2,59 \%$ e $4,97 \%$, respectivamente. O autor obteve também valores de $\Delta \mathrm{v}$ variando de $5,36 \%$ a $15,00 \%$.

Ainda, Vidaurre et al. (2018) ao avaliarem a madeira de paricá com idades variando de 5 a 11 anos, obtiveram valores de $\varepsilon l(0,34$ a $0,46 \%)$, er $(2,60$ a $3,54 \%)$, et $(4,98 \%$ a $5,48 \%)$ e $\Delta v(9,10$ a $9,60 \%)$ semelhantes aos do presente trabalho.

Por outro lado, os coeficientes de anisotropia, apresentados na Figura 5, possibilitaram indicar que as madeiras provenientes das classes intermediárias apresentam alto coeficiente de anisotropia (T/R > 2,0) indicando alta instabilidade dimensional (MARQUES et al., 2012). Porém, para as árvores das menores e das maiores classes diamétricas, foram observados valores de fator anisotrópico inferiores a 2,0.

Figura 5. Coeficientes de anisotropia (T/R) da madeira de paricá (Schizolobium parahyba var. amazonicum Huber ex Ducke) em função da classe diamétrica.

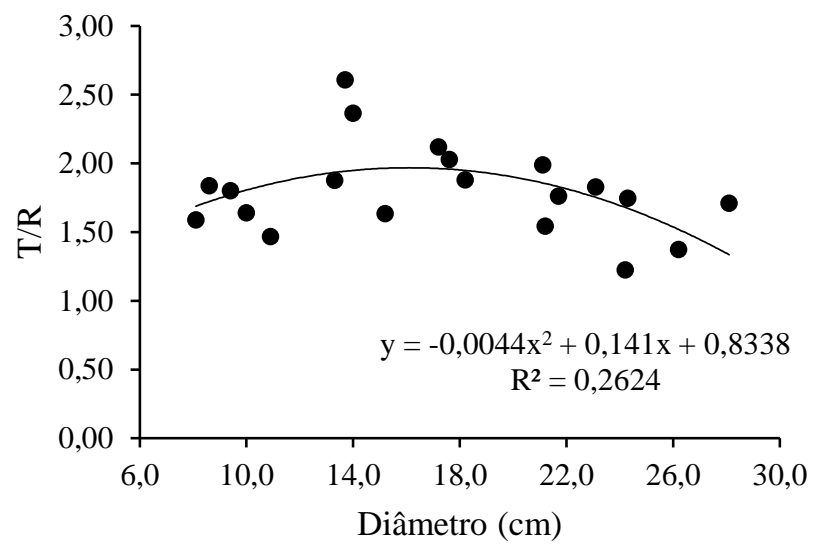

No entanto, de acordo com Melo et al. (2013), que também encontraram valores semelhantes ao do presente estudo, não se pode limitar o uso da madeira de paricá apenas com base nos coeficientes de anisotropia, pois tendo em vista as inúmeras técnicas existentes que visam a melhorar a estabilidade de madeiras e painéis de madeiras, como tratamentos termomecânicos, alteração química, laminação cruzada, preenchimento e revestimento, dentre outras.

O menores valores para $\mathrm{T} / \mathrm{R}$ foram observados para as classes IV e V, isto sugere que, devido ao maior diâmetro, tem-se maiores quantidades de lenho tardio, que apresentam paredes celulares mais espessas e menor teor de umidade que culminam em valores de contração tangencial e radial mais próximos e podem ser, ligeiramente, menores que aos encontrados em diâmetros inferiores (VIDAURRE et al., 2018), devido a relação inversamente proporcional com o coeficiente de anisotropia.

Nesse contexto, foi possível verificar que as propriedades físicas da madeira demonstraram poucas diferenças entre as classes diamétricas sendo que as classes que II e III são representadas pela maior frequência de árvores e que, em geral, apresentaram, por exemplo, boas características para confecção de compensados para diversas finalidades como, por exemplo, formas para concreto, andaimes, enquadramentos, bainhas, pisos, moldagem, postes, estacas, painéis, armários, móveis e instrumentos musicais (AZAMBUJA, 2015).

Dessa forma, diante dos aspectos abordados, mesmo que as diferenças observadas não tenham sido expressivas ao se avaliar as regressões, verificou-se que o estudo das propriedades tecnológicas da madeira podem subsidiar a ações realizadas no manejo de florestas plantadas, tendo em vista que a maioria dos trabalhos referentes ao desbaste seletivo e sortimento florestal são pautados em métodos quantitativos e não qualitativos, com isso, as propriedades físicas da madeira podem auxiliar na exploração do potencial de uso da madeira, direcionando-se as aplicações mais adequadas para esta matéria prima.

Os resultados obtidos podem contribuir para que as empresas relacionadas a produção madeireira possam fazer uso da madeira de paricá obtendo maior qualidade, facilitando o seu manejo, auxiliando na utilização adequada e aumento na produção com melhor direcionamento do produto.

\section{CONCLUSÃO}

Revista Desafios - v. 7, n. 3, 2020 
A madeira de paricá foi caracterizada como de baixa densidade, de alta porosidade e de boa estabilidade dimensional. $\mathrm{O}$ estudo das propriedades físicas permitiu identificar as diferenças entre os parâmetros físicos da madeira em função das classes diamétricas. Sugere-se que na realização de desbastes em povoamentos de paricá não se leve em consideração apenas os aspectos quantitativos, pois nem sempre as classes diamétricas mais frequentes e de maior volume vão apresentar características tecnológicas da madeira mais adequadas.

\section{AGRADECIMENTO}

Os autores agradecem aos proprietários da área de estudo, Lano da Amazônia LTDA, por possibilitarem a realização do presente trabalho.

Todos os autores declararam não haver qualquer potencial conflito de interesses referente a este artigo.

\section{REFERÊNCIAS}

ALVARES, A.C.; STAPE, J.L.; SENTELHAS, P.C.; MORAES, P.C.; GONÇALVES, J.L.; SPAROVEK, G. Köppen's climate classification map for Brazil. Meteorologische Zeitschrift, v. 22, n. 6, p.711-728, 2014.

AMERICAN SOCIETY FOR TESTING AND MATERIALS - ASTM. ASTM D143: Standard Test Methods for Small Clear Specimens of Timber. West Conshohocken: ASTM International, 2014.

AMERICAN SOCIETY FOR TESTING AND MATERIALS - ASTM. ASTM D5536: Standard Practice for Sampling Forest Trees for Determination of Clear Wood Properties. West Conshohocken: ASTM International, 2017.

AZAMBUJA, R.R. Aproveitamento de resíduos de construção e demolição para produção de painéis aglomerados. Dissertação de Mestrado. Universidade Federal do Paraná - UFPR; 2015.

CARVALHO, A. Madeiras portuguesas: estrutura anatómica, propriedades, utilizações. Lisboa, Instituto Florestal; 1996.
EUFRADE-JUNIOR, H.J.; BALLARIN, A.W.; VILLAMAGUA-VERGARA, G.C.; GUERRA, S.P.S. Efecto del manejo silvícola sobre la densidad básica de la madera en sistemas forestales de rotación corta. Maderas. Ciencia y tecnologia, v.19, n.3, p. 285-292, 2017.

INDÚSTRIA BRASILEIRA DE ÁRVORES - IBÁ. 2019. Relatório IBÁ 2019. Disponível em: https://iba.org/datafiles/publicacoes/relatorios/ibarelatorioanual2019.pdf

IWAKIRI, S.; TRIANOSKI, R.; NASCIMENTO, C.C.; JUIZO, C.G.F.; LEGOWSKI, E.C.; BILCATI, G.K.; GONÇALVES, T. Painéis aglomerados produzidos com seis espécies de madeiras tropicais da Amazônia. Madera y Bosques, v. 24, n. 3, e243371, 2018.

LABORATÓRIO DE PRODUTOS FLORESTAIS LPF. 2018. Banco de dados de espécies madeireiras brasileiras. Disponível em: http://sistemas.florestal.gov.br/madeirasdobrasil/fore word.htm

MARQUES, S.S.; OLIVEIRA, J.T.S.; PAES, J.B.; ALVES, E.S.; SILVA, A.G.; FIELDER, N.C. Estudo comparativo da massa específica aparente e retratibilidade da madeira de Pau-Brasil (Caesalpinia echinata Lam.) nativa e de reflorestamento. Revista Árvore, v. 36, n. 2, p. 373-380, 2012.

MASCARENHAS, A.R.P. Indicadores de qualidade ambiental e caracterização tecnológica da madeira em sistema agroflorestal multiestratificado. Dissertação de Mestrado. Universidade Federal de Rondônia UNIR; 2015.

MELO, L.E.L.; VASCONCELLOS, T.J.; SIVIERO, M.A.; CALLADO, C.H.; BARBOSA, A.C.M.C.; MORI, F.A. Axial variation in the cambium anatomy of Schizoloboium parahyba var. amazonicum. Holzforschung, v. 1, n. ahead-of-print, 2020.

MELO, L.E.L.; SILVA, C.J.; PROTÁSIO, T.P.; TRUGILHO, P.F.; SANTOS, I.S.; URBINATI C.V. Influência do espaçamento sobre algumas propriedades físicas da madeira de Schizolobium parahyba var. amazonicum (Huber ex Ducke). Scientia Forestalis, v. 42, n. 104, p. 483-490, 2014.

MELO, R. R.; DEL MENEZZI, C. H. S.; SOUZA, M. R., STANGERLIN, D. M. Avaliação das propriedades físicas, químicas, mecânicas e de superfície de lâminas de paricá (Schizolobium amazonicum Huber ex. Ducke). Floresta e Ambiente, v. 20 n. 2, p. 238-249, 2013. 
MELO, R. R.; STANGERLIN, D.M.; DEL MENEZZI, C.H.S.; GATTO, D.A.; CALEGARI, L. Caracterização física e mecânica da madeira de Araucaria angustifolia (Bertol.) Kuntze. Revista Brasileira de Ciências Agrárias, v. 5, n. 1, p. 67-73, 2010.

MIRANDA, D.L.C.; AMORIM, P.C.B.; SILVA, F.; LISBOA, G.S.; CONDÉ, T.M.; SILVA, C.S. Growth and production of paricá wood in two plantations in the north of Mato Grosso, Brazil. Nativa, v.4, n.4, p.199-205, 2016.

MOORE, J.R.; DASH, J.P.; LEE, J.R.; MCKINLEY, R.B.; DUNGEY, H.S. Quantifying the influence of seedlot and stand density on growth, wood properties and the economics of growing radiata pine. Forestry, v. 91, n. 1, p. $327-340,2018$.

MODES, K.S. Caracterização tecnológica da madeira de Schizolobium amazonicum Huber ex Ducke de florestas plantadas no estado de Rondônia. Tese de Doutorado. Universidade de São Paulo - USP; 2016.

MODES, K.S.; BORTOLETTO JÚNIOR, G.; TOMAZELLO FILHO, M.; SANTOS, L.M.H.; VIVIAN, M.A.; SILVA JÚNIOR, F.G. Caracterização morfológica e química da madeira de Schizolobium amazonicum aos 14 anos de plantios no estado de Rondônia. Scientia Forestalis, v. 46, n. 118, p. 271283, 2018.

RAMAGE, M.H.; BURRIDGE, H.; BUSSEWICHER, M.; FEREDAY, G.; REYNOLDS, T.; SHAH, D.U.; WU, G.; YU, L.; FLEMING, P.; DESLEY-TINGLEY, D.; ALLWOOD, J.; DUPREE, P.; LINDEN, P.D.; SCHERMAN, O. The wood from trees: The use of timber in construction. Renewable and Sustainable Energy Reviews, v.68, n.1, p.333359, 2017.

REIS, P.C.M.R.; REIS, L.P.; SOUZA, A.L.; CARVALHO, A.M.M.L.; MAZZEI, L.; REIS, A.R.S.; TORRES, C.M.M.E. Agrupamento de espécies madeireiras da Amazônia com base em propriedades físicas e mecânicas. Ciência Florestal, v. 29, n.1, p. 336-346, 2019.

ROMA, J.C.; ANDRADE, A.L.C. Economia, concessões florestais e a exploração sustentável de madeira. Boletim regional, urbano e ambiental do IPEA, v. 8, n. 1, p. 91-96, 2013.

SALES, A.; GONZÁLES, D.G.E.; MARTINS, T.G.V.; SILVA, G.C.C.; SPLETOZER, A.G.; TELLES, L.A.A.; SIVIERO, M.A.; LORENZON, A.S. Forests, v.10, n. 3, p. 252, 2019.

SILVA, C.B.R.; SANTOS JUNIOR, J.A.; ARAÚJO, A.J.C.; SALES, A.; SIVIERO, M.A.; ANDRADE, F.W.C.; CASTRO, J.P.; LATORRACA, J.V.F.; MELO, L.E.L. Agroforestry systems, v.94, n.1, p. 583-595, 2020.

SISTEMA DE PROTEÇÃO DA AMAZÔNIA SIPAM. Pedologia Rolim de Moura - RO: Mapa. Escala 1: 250.000. Porto Velho, Governo do Estado de Rondônia; 2006.

STEFANO, A.; BLAZIER, M.A.; COMER, C.E.; DEAN, T.J.; WIGLEY, T.B. Understory Vegetation Richness and Diversity of Eucalyptus benthamii and Pinus elliottii Plantations in the Midsouth US. Forest Science, p. 1-16, 2019.

TEREZO, R.F.; SZÜCS, C.A.; VALLE, A.; SAMPAIO, C.A.P.; STÜPP, A.M. Propriedades da madeira de paricá em diferentes idades para uso estrutural. Ciência da Madeira, v. 6, n. 3, p. 244-253, 2015.

TRUGILHO, P.F.; GOULART, S.L.; DE ASSIS, C.O.; COUTO, F.B.S.; ALVES, I.C.N.; PROTÁSIO, T.P.; NAPOLI, A. Características de crescimento, composição química, física e estimativa de massa seca de madeira em clones e espécies de Eucalyptus jovens. Ciência Rural, v. 45, n. 4, p. 661-666, 2014.

VERÍSSIMO, A.; PEREIRA, D. Produção na Amazônia Florestal: características, desafios e oportunidades. Parcerias Estratégicas, v. 19, n. 38, p. 13-44, 2014.

VIDAURRE, G.B.; VITAL, B.R.; OLIVEIRA, A.D.C.; OLIVEIRA, J.T.D.S.; MOULIN, J.C.; SILVA, J.G.M.D.; SORANSO, D.R. Physical and mechanical properties of juvenile Schizolobium amazonicum wood. Revista Árvore, v. 42, n. 1, p. 29, 2018. 